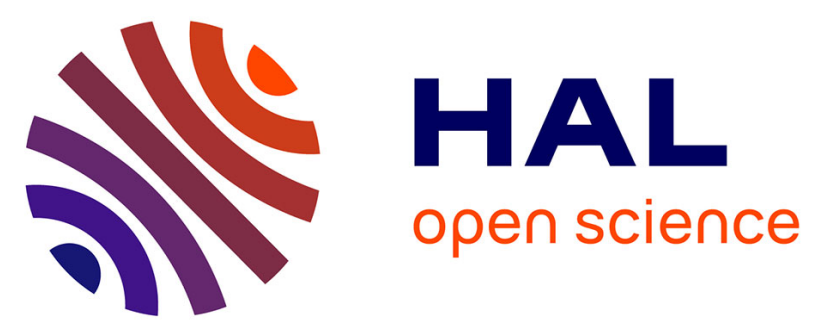

\title{
Effect of the porosity on the fracture surface roughness of sintered materials: From anisotropic to isotropic self-affine scaling
}

Tristan Cambonie, Jonathan Barés, M. L. Hattali, Daniel Bonamy, Veronique Lazarus, Harold Auradou

\section{To cite this version:}

Tristan Cambonie, Jonathan Barés, M. L. Hattali, Daniel Bonamy, Veronique Lazarus, et al.. Effect of the porosity on the fracture surface roughness of sintered materials: From anisotropic to isotropic self-affine scaling. Physical Review E: Statistical, Nonlinear, and Soft Matter Physics, 2015, 91 (1), pp.012406. 10.1103/PhysRevE.91.012406 . cea-01366743

\section{HAL Id: cea-01366743 https://hal-cea.archives-ouvertes.fr/cea-01366743}

Submitted on 15 Sep 2016

HAL is a multi-disciplinary open access archive for the deposit and dissemination of scientific research documents, whether they are published or not. The documents may come from teaching and research institutions in France or abroad, or from public or private research centers.
L'archive ouverte pluridisciplinaire HAL, est destinée au dépôt et à la diffusion de documents scientifiques de niveau recherche, publiés ou non, émanant des établissements d'enseignement et de recherche français ou étrangers, des laboratoires publics ou privés. 


\title{
Effect of the porosity on the fracture surface roughness of sintered materials: From anisotropic to isotropic self-affine scaling
}

\author{
T. Cambonie,,${ }^{1, *}$ J. Bares, ${ }^{2, \dagger}$ M. L. Hattali, ${ }^{1}$ D. Bonamy,,${ }^{2, \dagger}$ V. Lazarus, ${ }^{1}$ and H. Auradou ${ }^{1}$ \\ ${ }^{1}$ Université Paris-Sud, CNRS, UMR 7608, Laboratoire FAST, Bat. 502, Campus Université, F-91405 Orsay, France \\ ${ }^{2}$ CEA, IRAMIS, SPEC, SPHYNX Laboratory, F-91191 Gif sur Yvette, France
}

(Received 1 September 2014; published 16 January 2015)

\begin{abstract}
To unravel how the microstructure affects the fracture surface roughness in heterogeneous brittle solids like rocks or ceramics, we characterized the roughness statistics of postmortem fracture surfaces in homemade materials of adjustable microstructure length scale and porosity, obtained by sintering monodisperse polystyrene beads. Beyond the characteristic size of disorder, the roughness profiles are found to exhibit self-affine scaling features evolving with porosity. Starting from a null value and increasing the porosity, we quantitatively modify the self-affine scaling properties from anisotropic (at low porosity) to isotropic (for porosity $>10 \%$ ).
\end{abstract}

DOI: 10.1103/PhysRevE.91.012406

PACS number(s): $46.50 .+\mathrm{a}$

\section{INTRODUCTION}

Fractography, i.e., the morphological characterization of postmortem fracture surfaces, is a classical tool used to identify the mechanisms and the damaging processes (fatigue, stress corrosion, cleavage, plastic cavitation, crazing, etc.) responsible for failure (see [1] for a recent textbook). Since the pioneer work of Mandelbrot et al. [2], numerous studies have evidenced the existence of self-affine scaling invariance over a wide range of length scales (see [3,4] for reviews). Early measurements in various materials including metallic alloys, ceramics, and rocks [5-7] reported values for the roughness exponent $\zeta$ close to 0.8 , suggesting the existence of a universal value $[5,6]$, independent of the propagation mode and material. These experimental observations yielded intense theoretical [8-14] and numerical [15-18] research.

Recently, the picture has been made more complex. First, the roughness exponent has been found to depend (slightly) on the anisotropy of the material microstructure [19] and on the fracture speed [20]. Second, fracture surfaces were shown to exhibit anisotropic scaling features, characterized by two roughness exponents whether observed along the direction of the crack front or along the direction of crack growth $[21,22]$. Third, fracture surfaces exhibit anomalous scaling [23,24]; the introduction of an additional global roughness exponent $\zeta_{\text {glob }}$ is then necessary to describe the scaling between the global crack width and the specimen size. Fourth, multiaffinity, disappearing at large scales, was invoked [25,26]. Finally, the scale invariance properties were found to depend on the propagation mode: The seminal self-affine feature with $\zeta \simeq$ 0.8 is to be linked with quasibrittle fracture; lower values for $\zeta$, around 0.4-0.5 [27-30] or even logarithmic roughness [31], are to be associated with brittle fracture; and a multiaffine regime with a roughness exponent close to 0.5 is characteristic of ductile fracture [32-34]. Note that different regimes can be observed on the same fracture surface $[14,35,36]$, depending on the scale of observation and its position with respect

\footnotetext{
*cambonie@fast.u-psud.fr

${ }^{\dagger}$ Present address: Duke University, Durham, North Carolina 27708, USA.

${ }^{\ddagger}$ daniel.bonamy@cea.fr
}

to the relevant sizes associated with the various dissipation mechanisms (plastic zone size, damage zone size, fracture process zone size). This enables us to infer the fracture process zone from a statistical characterization of the crack roughness [37].

Most past studies dedicated to the roughness of cracks aimed at characterizing the scale-invariant properties of fracture surfaces. This quest for universality classes, i.e., features independent of the fracturing conditions (loading and material parameters), strayed from the initial metrologic purpose of the fractography science. Here, we go back to this primary purpose and seek to characterize how the microstructure affects the fracture surface roughness. Rather than using existing materials like ceramics or rocks, we use homemade porous solids obtained by sintering spherical monodisperse grains. The advantage is that both the grain size and the porosity can be easily adjusted by modifying, respectively, the bead diameter and the sintering parameters, while presenting a structure of cemented grains very comparable to the structures found in natural heterogeneous brittle solids like rocks (sandstones, for instance) and concrete and other artificial heterogeneous brittle solids like pharmaceutical pills and sintered ceramics. The procedure for preparing the samples is described in detail in Sec. II A. The samples are finally broken using a technique detailled in Sec. II B that permits us to grow stable mode I cracks. The fractography of the surfaces (see Sec. II C) is used to determine the propagation mode of the fracture, i.e., interor intragranular propagation. The spatial correlation of the roughness of the crack surfaces is, finally, characterized by the structure function of profiles taken along and perpendicular to the direction of crack propagation. The evolution of this function as a function of the bead size, the direction of measurements, and the porosity is reported in Sec.III. Finally, in Sec. IV we discuss the change in the self-affine exponents observed when the porosity is modified.

\section{SAMPLE PREPARATION AND EXPERIMENTAL SETUP}

The experiments reported here consist (i) in driving a stable tensile crack with a controlled velocity in a material obtained by sintering polystyrene beads and (ii) subsequently, in analyzing the spatial distribution of the crack roughness. The material 


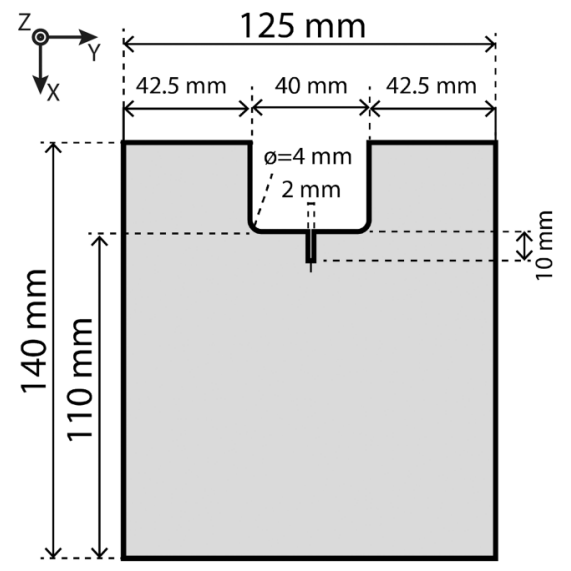

(a)

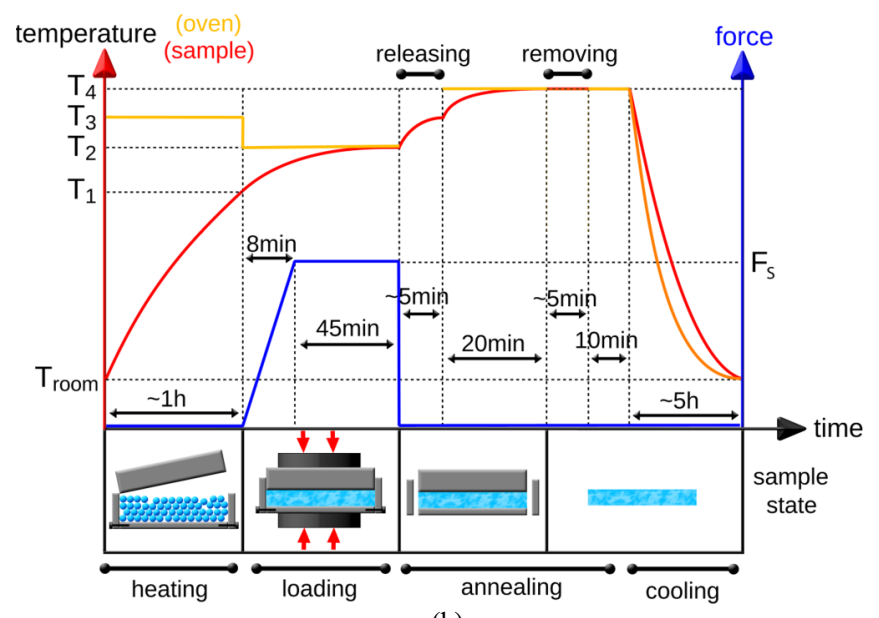

(b)

\begin{tabular}{|c|c|c|c|c|c|}
\hline$d_{0}$ & $21 \mu \mathrm{m}$ & $42 \mu \mathrm{m}$ & $81 \mu \mathrm{m}$ & $228 \mu \mathrm{m}$ & $583 \mu \mathrm{m}$ \\
\hline$T_{1}\left({ }^{\circ} \mathrm{C}\right)$ & 90 & 90 & 105 & 105 & 105 \\
\hline$T_{2}\left({ }^{\circ} \mathrm{C}\right)$ & 100 & 100 & 115 & 115 & 115 \\
\hline$T_{3}\left({ }^{\circ} \mathrm{C}\right)$ & 105 & 115 & 120 & 120 & 120 \\
\hline$T_{4}\left({ }^{\circ} \mathrm{C}\right)$ & 110 & 120 & 130 & 130 & 130 \\
\hline
\end{tabular}

(c)

FIG. 1. (Color online) (a) Geometry and dimensions of the wedge-splitting samples. (b) Evolution sketch of the temperature of the oven, of the sample, and of the force applied during the sintering protocol (see [38] for details). (c) Temperature values at the different stages of the sintering protocol for the different bead sizes.

fabrication, fracture setup, and topographical recording of the postmortem roughness profiles are presented thereafter.

\section{A. Sintering protocol}

Samples are obtained by sintering monodisperse polystyrene beads (Dynoseeds) of various diameters. Four steps are achieved: (i) a homemade mold, whose geometry is given in Fig. 1(a), is filled with beads and heated up to $90 \%$ of the temperature at glass transition to soften the beads; (ii) a slowly linearly increasing compressive force is then applied up to the prescribed sintering load $F_{s}$ for nearly 1 $\mathrm{h}$ to achieve sintering; (iii) an annealing stage is performed, in which the mold is unloaded, unscrewed, and loosened, keeping the temperature high enough to avoid thermal shocks; and (iv) the sample is finally slowly cooled down to ambient
TABLE I. Values of experimental parameters associated with the different fracture surfaces analyzed here: bead diameter $d_{0}$ prior to sintering, sintering force $F_{s}$, wedge velocity $V_{\text {wdg }}$, and porosity $\Phi$.

\begin{tabular}{|c|c|c|c|c|c|c|}
\hline & \multicolumn{6}{|c|}{ Experiment No. } \\
\hline & 1 & 2 & 3 & 4 & 5 & 6 \\
\hline$d_{0}(\mu \mathrm{m})$ & 21 & 21 & 42 & 42 & 42 & 81 \\
\hline$F_{S}(\mathrm{kN})$ & 4 & 8 & 1 & 8 & 8 & 2 \\
\hline$V_{\mathrm{wdg}}\left(\mathrm{nm} \mathrm{s}^{-1}\right)$ & 16 & 16 & 16 & 16 & 16 & 16 \\
\hline \multirow[t]{3}{*}{$\Phi(\%)$} & 1 & 0 & 1 & 0 & 0 & 1 \\
\hline & \multicolumn{6}{|c|}{ Experiment No. } \\
\hline & 7 & 8 & 9 & 10 & 11 & 12 \\
\hline$\overline{d_{0}(\mu \mathrm{m})}$ & 81 & 81 & 81 & 81 & 228 & 228 \\
\hline$F_{S}(\mathrm{kN})$ & 4 & 8 & 8 & 8 & 2 & 4 \\
\hline$V_{\mathrm{wdg}}\left(\mathrm{nm} \mathrm{s}^{-1}\right)$ & 16 & 16 & 16 & 16 & 16 & 16 \\
\hline \multirow[t]{3}{*}{$\Phi(\%)$} & 1 & 0 & 0 & 0 & 1 & 0 \\
\hline & \multicolumn{6}{|c|}{ Experiment No. } \\
\hline & 13 & 14 & 15 & 16 & 17 & \\
\hline$\overline{d_{0}(\mu \mathrm{m})}$ & 228 & 583 & 583 & 583 & 583 & \\
\hline$F_{\mathrm{s}}(\mathrm{kN})$ & 8 & 8 & 8 & 8 & 8 & \\
\hline$V_{\mathrm{wdg}}\left(\mathrm{nm} \mathrm{s}^{-1}\right)$ & 16 & 1.6 & 16 & 160 & 1600 & \\
\hline \multirow[t]{3}{*}{$\Phi(\%)$} & 0 & 0 & 0 & 0 & 0 & \\
\hline & \multicolumn{6}{|c|}{ Experiment No. } \\
\hline & 18 & 19 & 20 & 21 & 22 & 23 \\
\hline$\overline{d_{0}(\mu \mathrm{m})}$ & 21 & 81 & 42 & 42 & 42 & 42 \\
\hline$F_{S}(\mathrm{kN})$ & 0.1 & 0.1 & 0.1 & 0.2 & 0.6 & 0.8 \\
\hline$V_{\text {wdg }}\left(\mathrm{nm} \mathrm{s}^{-1}\right)$ & 16 & 16 & 16 & 16 & 16 & 16 \\
\hline$\Phi(\%)$ & 11.65 & 18.7 & 15.1 & 10.4 & $\approx 3$ & $\approx 2$ \\
\hline
\end{tabular}

temperature. The whole sintering protocol is sketched with more details in Fig. 1(b), and the relevant temperatures are listed in Fig. 1(c) (see also [38] for an extensive presentation).

The bead diameter $d_{0}$ was varied between 21 and $583 \mu \mathrm{m}$. By changing $F_{\mathrm{s}}$ between 0.1 and $8 \mathrm{kN}$ (corresponding to a loading pressure ranging from 6 to $490 \mathrm{kPa}$ ), we are able to fabricate specimens of porosities between $\sim 0 \%$ and $19 \%$ (see Table I). Smaller values of $F_{s}$ lead to unusable friable samples. The porosity $\Phi$ is measured as the ratio $\Phi=1-$ $\rho / \rho_{0}$ of the density of the sample $\rho$ and a reference density $\rho_{0}$ corresponding to the density of the void-free samples obtained with the highest sintering force. It is found that $\rho_{0}$ is slightly bead size dependent, which may be explained by the slightly different initial bead material.

\section{B. Fracture wedge-splitting tests}

Specimens [Fig. 1(a)] are parallelepipeds of dimension $140 \times 125 \times W \mathrm{~mm}^{3}$ in the $x \times y \times z$ direction, where the specimen width $W$ depends on the material porosity and ranges from $15 \mathrm{~mm}$ (for the most compacted specimens) to $20 \mathrm{~mm}$ (for the most porous specimens). A rectangular notch is milled by cutting a $42 \times 30 \times W \mathrm{~mm}^{3}$ rectangle from the sample at one of the two lateral faces. A 10-mm-long, 2-mm-thick groove is then introduced in the middle of the rectangular notch using a diamond saw, and a seed precrack is added at the groove tip by means of a razor blade. The latter operation prevents the propagation of a dynamic fracture and allows us to grow slow stable cracks. Thereafter, we define the axes $x$ parallel to the direction of crack propagation, $y$ perpendicular to the mean crack plane, and $z$ parallel to the mean crack front. 


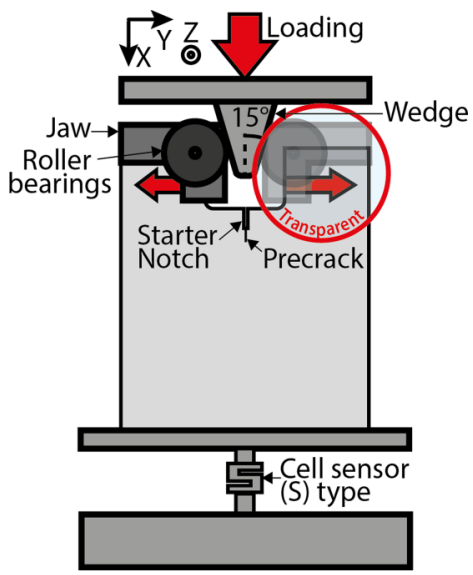

(a)

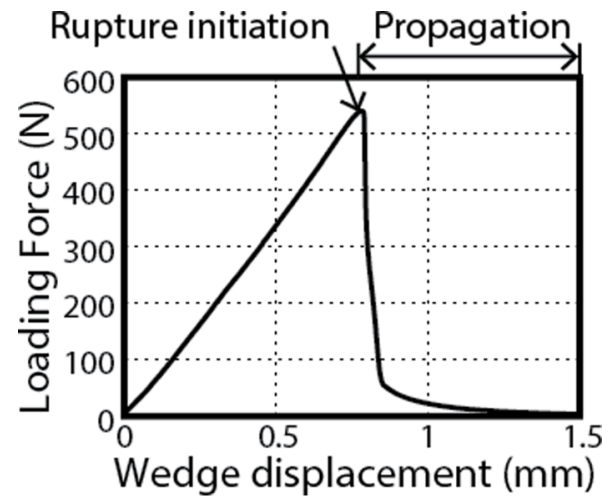

(b)

FIG. 2. (Color online) (a) Sketch of the experimental setup. (b) Typical force-vs-displacement curve.

The sample is then placed between the two jaws of a loading machine (a homemade or a commercial electromechanical machine). A homemade metallic wedge (semiangle of $15^{\circ}$ ) is placed between the jaw and the specimen notch [Fig. 2(a)]. It has been designed to minimize friction dissipation by means of two rollers and to convert the compression along $x$ into a tension along $y[39,40]$. The sample is then compressed by lowering the upper jaw at a constant velocity. In the experiments reported here, the wedge speed $V_{\mathrm{wdg}}$ varied from 1.6 to $1600 \mathrm{~nm} \mathrm{~s}^{-1}$. During each test, the force $F(t)$ applied by the wedge to the specimen was monitored in real time by a static load cell. As soon as the wedge starts to push on the specimen, $F$ increases. When $F(t)$ reaches the critical loading [Fig. 2(b)], the crack starts to propagate. Prior to fracture the load-displacement curve is linear, confirming the elastic behavior of the material. The crack velocity is observed first to increase (over $\sim 20 \mathrm{~mm}$ ), then to stabilize at a roughly constant value, $\sim 100$ times the wedge speed $V_{\text {wdg }}$ (over 40 to $60 \mathrm{~mm}$ ), and, finally, to decrease over the last $20 \mathrm{~mm}$. Our study focuses on the interval in which the velocity is constant and all measurements are done in that region.

\section{Postmortem analysis of crack surfaces}

Once the samples were broken into two halves, the morphology of the postmortem crack surfaces was analyzed. First, the surfaces were visualized by means of a Keyence numerical microscope. This microscope automatically performs a vertical scan at different heights and reconstructs a well-focused image pixel by pixel over the whole topography range, irrespective of the level of height variations.

Topography profiles are also recorded by a Bruker mechanical profilometer. Three profiles are scanned along the $x$ direction and three others along the $z$ direction. Their lengths are respectively $55 \mathrm{~mm}$ along $x$ and $14 \mathrm{~mm}$ along $z$. Each profile has been positioned in the central part of the fracture surface away from the sample borders. They are located between $x=40 \mathrm{~mm}$ and $x=95 \mathrm{~mm}$ and at $z=\{-3,0,3\} \mathrm{mm}$ for profiles in the $x$ direction and between $z=-7$ and $z=7$ $\mathrm{mm}$ and at $x=\{-40,60,80\} \mathrm{mm}$ for profiles in the $z$ direction. The out-of-plane and in-plane resolutions are $\delta y=0.1 \mathrm{~nm}$ and $\delta x=\delta z=0.8 \mu \mathrm{m}$, which corresponds, per profile, to 68750 points in the $x$ direction and 16250 in the $z$ direction.

For $d_{0}=583 \mu \mathrm{m}$, the fracture surfaces have been measured using another profilometer due to the vertical displacement limitation of the Bruker profilometer [30]. Its out-of-plane and in-plane resolutions are $\delta y=100 \mathrm{~nm}$ and $\delta x=\delta z=1 \mu \mathrm{m}$. To have a better statistic, ten $75-\mathrm{mm}$-long profiles have been measured in the $x$ direction. Yet, in this case, due to the small number of beads in the thickness, no measurements were made in the $z$ direction.

\section{RESULTS}

A general overview of the fracture surfaces obtained by changing the bead size, the porosity, and the crack velocity is first given. In most cases, the propagation is intergranular. We focus on this case, investigating the effects of the microstructure size and porosity on the fracture surfaces.

\section{A. Fractography}

Figure 3 presents microscope visualizations of typical fracture surfaces, for different bead diameters, porosities, and wedge velocities. The crack propagates from left to right.

Figures 3(a)-3(c) correspond to a null porosity and to samples broken at the same wedge velocity but with different bead diameters. The particle surfaces are observed to be completely deformed, resulting in sharp edges and corners, in agreement with the zero value of the porosity. Also, the surfaces are found to be made of smooth facets. Such a faceted morphology is characteristic of a brittle intergranular fracture, with a crack growing along the grain-grain interfaces. Note that the facets are slightly more elongated along $x$ than along $z$ [visible in Fig. 3(b)]. This anisotropy is due to the sintering process. In fact, the compression was applied along $z$, or in a granular packing, the two components $\sigma_{x x}$ and $\sigma_{z z}$ of the stress tensor are classically related [41] via $\sigma_{x x}=$ $K \sigma_{z z}$, where the Janssen constant $K$ is less than 1, typically around 0.6-0.8. During sintering, the beads, hence, contract more along $z$ than along $x$. Figure 3(d), corresponding to a lower wedge speed, $V_{\text {wdg }}=1.6 \mathrm{~nm} / \mathrm{s}$, presents a qualitatively different morphology: The facets are observed to be blurred, with a multitude of small-scale fragments. They betray an intragranular propagation mode, with a crack propagating throughout the grains, without necessarily following the graingrain interfaces. 


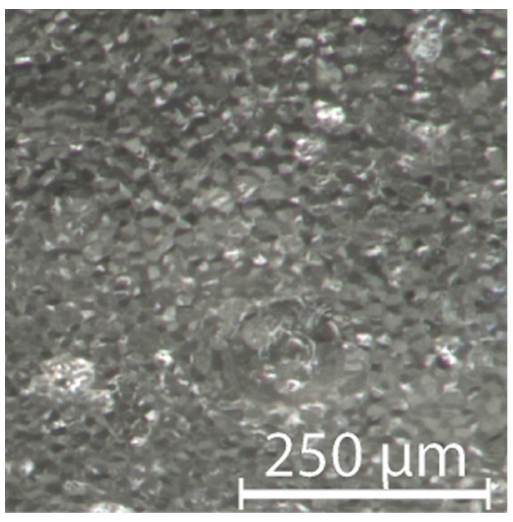

(a)

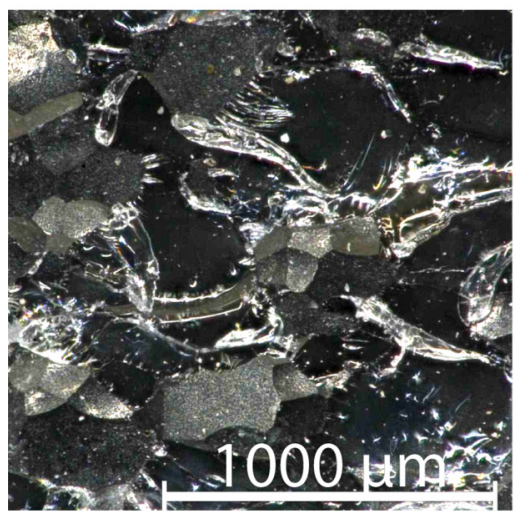

(d)

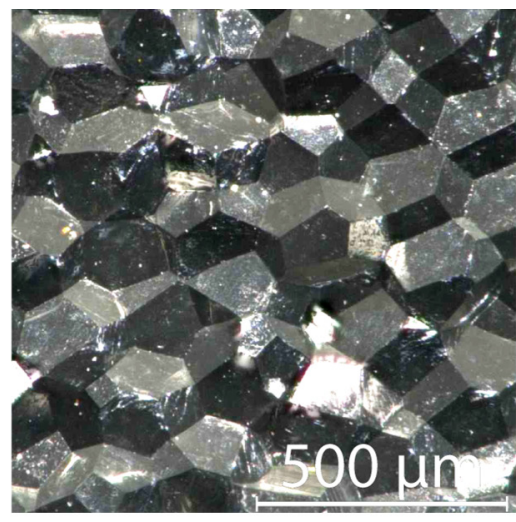

(b)

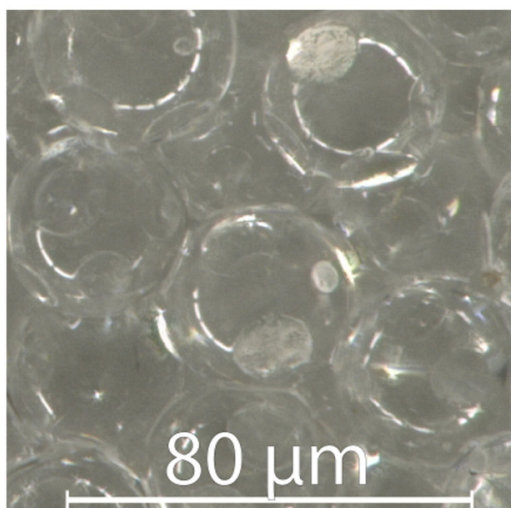

(e)

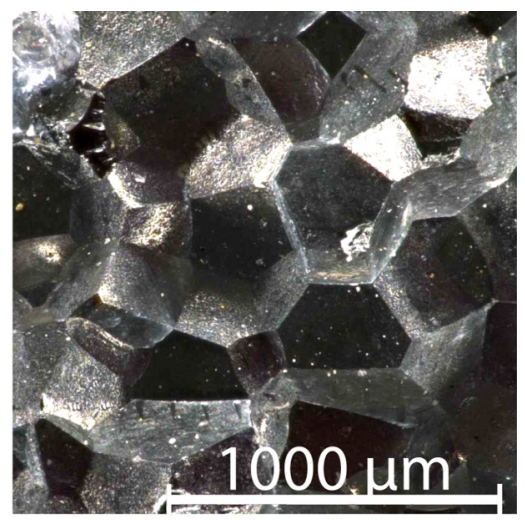

(c)

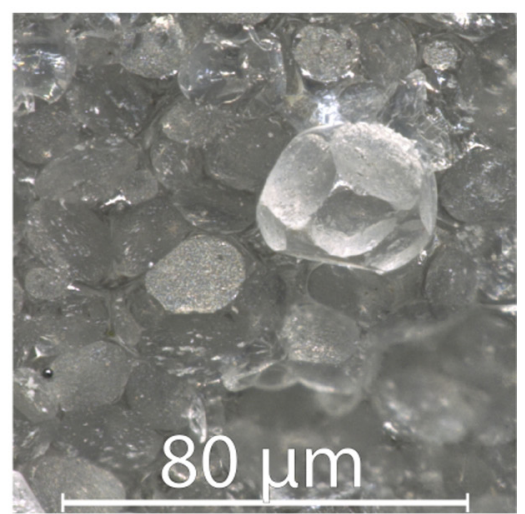

(f)

FIG. 3. (Color online) Microscope visualization of wedge-split fractured surfaces of the sintered-PS-bead samples. The bead diameter $d_{0}$, wedge speed $V_{\text {wdg }}$, sintering load $F_{s}$, and corresponding porosity $\Phi$ are as follows.

(a) $d_{0}=21 \mu \mathrm{m}, V_{\mathrm{wdg}}=16 \mathrm{~nm} \mathrm{~s}^{-1}, F_{s}=8 \mathrm{t}, \Phi=0 \%$.

(b) $d_{0}=228 \mu \mathrm{m}, V_{\mathrm{wdg}}=16 \mathrm{~nm} \mathrm{~s}^{-1}, F_{s}=8 \mathrm{t}, \Phi=0 \%$.

(c) $d_{0}=583 \mu \mathrm{m}, V_{\mathrm{wdg}}=16 \mathrm{~nm} \mathrm{~s}^{-1}, F_{\mathrm{s}}=8 \mathrm{t}, \Phi=0 \%$.

(d) $d_{0}=583 \mu \mathrm{m}, V_{\mathrm{wdg}}=1.6 \mathrm{~nm} \mathrm{~s}^{-1}, F_{s}=8 \mathrm{t}, \Phi=0 \%$.

(e) $d_{0}=42 \mu \mathrm{m}, V_{\text {wdg }}=16 \mathrm{~nm} \mathrm{~s}^{-1}, F_{s}=0.1 \mathrm{t}, \Phi=15 \%$.

(f) $d_{0}=42 \mu \mathrm{m}, V_{\text {wdg }}=16 \mathrm{~nm} \mathrm{~s}^{-1}, F_{s}=1 \mathrm{t}, \Phi=1.45 \%$.

Figures 3(e) and 3(f) show microscopic visualizations of fracture surfaces of sintered samples realized with a lower sintering force $F_{\mathrm{s}}$. At low $F_{\mathrm{s}}$ [Fig. 3(e)], the beads globally maintain a spheric shape. The contact areas between adjacent beads are small and disk shaped. As a result, an important porosity is found in such samples. When $F_{\mathrm{s}}$ gets larger, the contact surfaces grow and intersect, resulting in the appearance of sharp edges of increasing length [Fig. 3(f)], while the undeformed spherical parts of the particles shrink, so that the volume of the pores, delimited by these parts, decreases. When $F_{\mathrm{s}}$ is large enough to close all the pores [Fig. 3(c)], the undeformed parts of the particles disappear and the corners are sharp.

Figure 4 presents typical topographical profiles $H(z)$ as measured along the $z$ direction on the fracture surfaces shown in Fig. 3. In Figs. 4(a)-4(c), associated with intergranular fracture, the corrugations in the $y$ and $z$ directions are of the same order, that of the bead size. Conversely, the roughness observed for the intragranular mode [Fig. 4(d)] appears much flatter and exhibits plateaus much longer than the bead diameter. These plateaus correspond to zones where the crack has cut throughout the grains, without being perturbed by the interfaces. Finally, Figs. 4(e) and 4(f) show typical profiles at finite porosity. Note the increase in roughness with amplitudes higher than the bead diameter $d_{0}$. The shift from inter- to intragranular fractures occurs for a fracture velocity of 1.6 $\mathrm{nm} / \mathrm{s}$. In the present study, we focus on the intergranular case, hence on experiments corresponding to $V_{\mathrm{wdg}}>1.6 \mathrm{~nm} / \mathrm{s}$. Works on the transition between intra- and intergranular propagation and on intragranular fracturation are in progress.

\section{B. Effect of the microstructure length scale}

We first restrict the analysis to the effect of the microstructure length scale and, hence, only consider specimens (nos. $1-17$ in Table I) with a very low porosity $(<0.5 \%)$. To quantify the spatial distribution of the crack roughness, we computed the structure functions [42] $S_{z}(\Delta z)$ along $z$ and $S_{x}(\Delta x)$ along $x$, defined by

$$
\begin{aligned}
S_{z}(\Delta z) & =\left\langle(H(z+\Delta z)-H(z))^{2}\right\rangle, \\
S_{x}(\Delta x) & =\left\langle(H(x+\Delta x)-H(x))^{2}\right\rangle,
\end{aligned}
$$




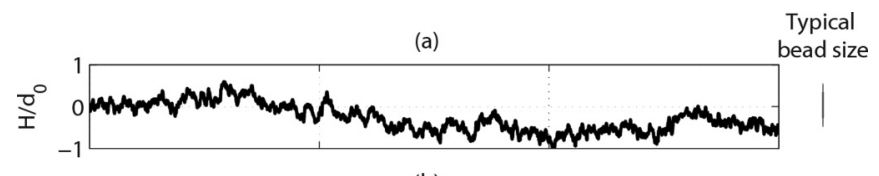

(b)

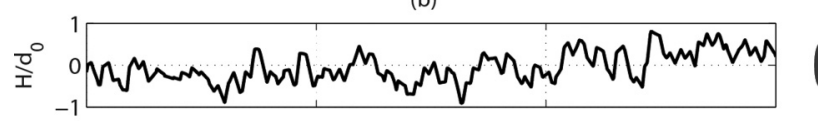

(c)

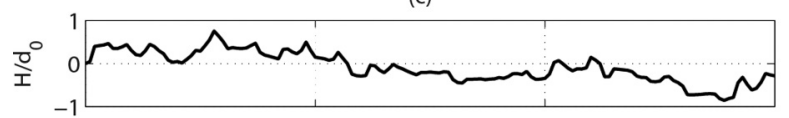

(d)

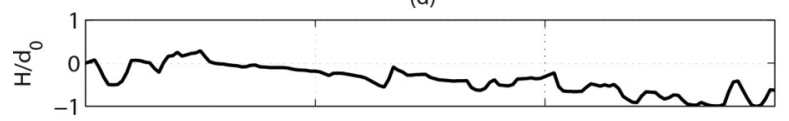

(e)

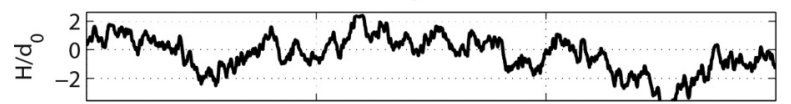

(f)

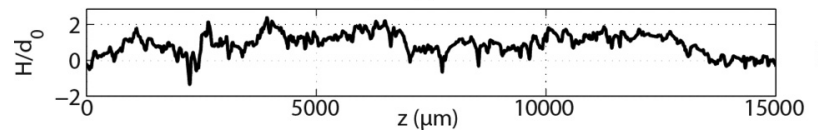

FIG. 4. Experimental height profiles along the crack propagation direction of the same samples as in Fig. 3. At the right, the bead shape is given as a reference, using the same abscissa and ordinate scales as in the plots. Since these scales are different, the bead appears distorted. (a) Experiment 2: $d_{0}=21 \mu \mathrm{m}, V_{\mathrm{wdg}}=16 \mathrm{~nm} \mathrm{~s}^{-1}, \Phi=0 \%$. (b) Experiment 13: $d_{0}=228 \mu \mathrm{m}, V_{\mathrm{wdg}}=16 \mathrm{~nm} \mathrm{~s}^{-1}, \Phi=0 \%$. (c) Experiment 16: $d_{0}=583 \mu \mathrm{m}, V_{\mathrm{wdg}}=16 \mathrm{~nm} \mathrm{~s}^{-1}, \Phi=0 \%$. (d) Experiment 14: $d_{0}=583 \mu \mathrm{m}, V_{\mathrm{wdg}}=1.6 \mathrm{~nm} \mathrm{~s}^{-1}, \Phi=0 \%$. (e) Experiment 21: $d_{0}=42 \mu \mathrm{m}, V_{\mathrm{wdg}}=16 \mathrm{~nm} \mathrm{~s}^{-1}, \Phi=15 \%$. (f) Experiment 3: $d_{0}=42 \mu \mathrm{m}, V_{\mathrm{wdg}}=16 \mathrm{~nm} \mathrm{~s}^{-1}, \Phi=1 \%$

where the operator \langle\rangle refers to an average over all possible positions $z$ and $x$, respectively.

Figure 5 displays in a log-log plot the structure functions $S_{x}$ measured along $x$ for different bead diameters. Two scaling regimes can be distinguished. At large scales $(\Delta x \gg 10 \mu \mathrm{m})$, $S_{x}$ slowly increases with $\Delta x$, with a prefactor increasing with $d_{0}$. This betrays the fact that the roughness scales with $d_{0}$. At small scales $(\Delta x \ll 10 \mu \mathrm{m})$, all curves collapse, and the roughness in this regime is weakly dependent on $d_{0}$.

The diameter $d_{0}$ appears to be the only length scale in the problem. Therefore, we made dimensionless the structure functions by making $\Delta x \rightarrow \Delta x / d_{0}$ and $S_{x} \rightarrow S_{x} / d_{0}^{2}$ (Fig. 5, inset). A good collapse is obtained, confirming that $d_{0}$ is the length scale governing the spatial distribution of the roughness.

Nevertheless, the collapse shown in the inset in Fig. 5 is not perfect and a clear scattering remains visible. We applied the global minimization technique described in Ref. [43] to reduce the dispersion of the data. For each curve, an optimal length $\ell$ is obtained. The values are listed in Table II for each sample. Figures 6(a) and 6(b) show the structure functions in the $x$ and $z$ directions normalized by $\ell_{x}$ and $\ell_{z}$. As shown, the sole parameters $\ell_{x}$ and $\ell_{z}$ are sufficient to achieve a very good collapse. We checked that these lengths correspond to the microstructure sizes in both directions (as, e.g., shown in

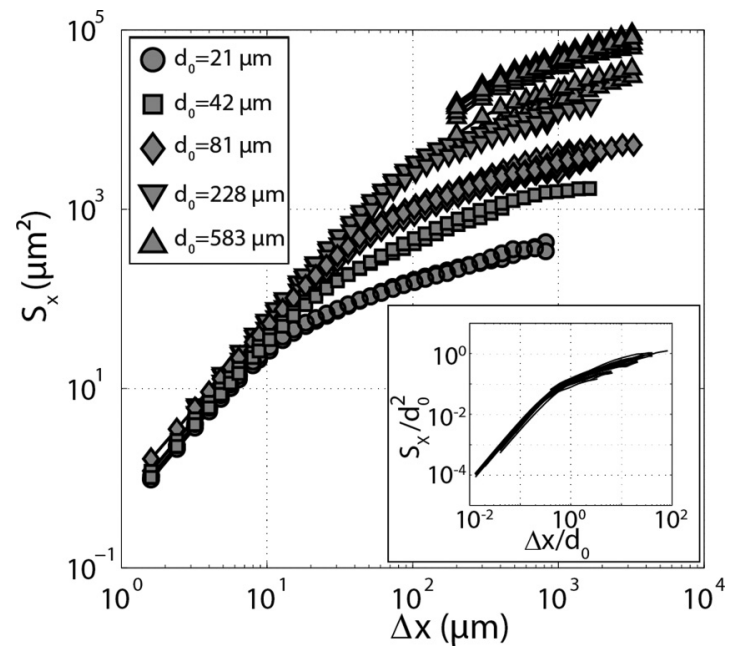

FIG. 5. Structure functions $S_{x}$ of intergranular experiments, along the propagation direction at $\Phi=0 \%$. Each symbol corresponds to a given value of $d_{0}$. Inset: Same distributions made dimensionless by $d_{0}^{2}$.

Fig. 3). In other words, the slight differences observed among $\ell_{x}, \ell_{z}$, and $d_{0}$ result from the sintering process.

Moreover, two power-law regimes, characterized by two different exponents, are observed. To determine these exponents, we performed linear fits of the data displayed in Fig. 6. The roughness exponents $\zeta_{x}^{+,-}$and $\zeta_{z}^{+,-}$, for length scales above and below $d_{0}$, are defined by the relations $S_{x} \propto \Delta x^{2 \zeta_{x}}$ and $S_{z} \propto \Delta z^{2 \zeta_{z}}$. Table III lists the roughness exponent values along the propagation and front crack directions. In the small-scale regime, the exponents $\zeta^{-}$are close to 0.8 in both directions. At larger scales, the exponents $\zeta^{+}$are functions of the direction of measurement, the roughness along the crack being more important than that in the propagation direction. Altough the exponents are relatively small with respect to usual

TABLE II. Optimal lengths $\ell_{x}$ and $\ell_{z}$ along the $x$ and $z$ directions used to collapse (in Fig. 6 ) the structure functions.

\begin{tabular}{|c|c|c|c|c|c|c|}
\hline & \multicolumn{6}{|c|}{ Experiment No. } \\
\hline & 1 & 2 & 3 & 4 & 5 & 6 \\
\hline$d_{0}(\mu \mathrm{m})$ & 21 & 21 & 42 & 42 & 42 & 81 \\
\hline$\Phi(\%)$ & 1 & 0 & 1 & 0 & 0 & 1 \\
\hline$\ell_{z}(\mu \mathrm{m})$ & 19.8 & 19.6 & 19.6 & 39.9 & 44 & 79.1 \\
\hline \multirow[t]{3}{*}{$\ell_{x}(\mu \mathrm{m})$} & 20.2 & 21.8 & 26.4 & 44.8 & 44.9 & 81.7 \\
\hline & \multicolumn{6}{|c|}{ Experiment No. } \\
\hline & 7 & 8 & 9 & 10 & 11 & 12 \\
\hline$\overline{d_{0}(\mu \mathrm{m})}$ & 81 & 81 & 81 & 81 & 228 & 228 \\
\hline$\Phi(\%)$ & 1 & 0 & 0 & 0 & 1 & 0 \\
\hline$\ell_{z}(\mu \mathrm{m})$ & 70.2 & 80.3 & 68.4 & 76 & 203.3 & 187.4 \\
\hline \multirow{3}{*}{$\ell_{x}(\mu \mathrm{m})$} & 74.8 & 82.9 & 81.7 & 83.7 & 238.7 & 229.2 \\
\hline & \multicolumn{6}{|c|}{ Experiment No. } \\
\hline & 13 & 14 & 15 & 16 & 17 & \\
\hline$\overline{d_{0}(\mu \mathrm{m})}$ & 228 & $x$ & 583 & 583 & 583 & \\
\hline$\Phi(\%)$ & 0 & $x$ & 0 & 0 & 0 & \\
\hline$\ell_{z}(\mu \mathrm{m})$ & 166 & $x$ & $x$ & $x$ & $x$ & \\
\hline$\ell_{x}(\mu \mathrm{m})$ & 192.9 & $x$ & 381 & 486.5 & 440.8 & \\
\hline
\end{tabular}




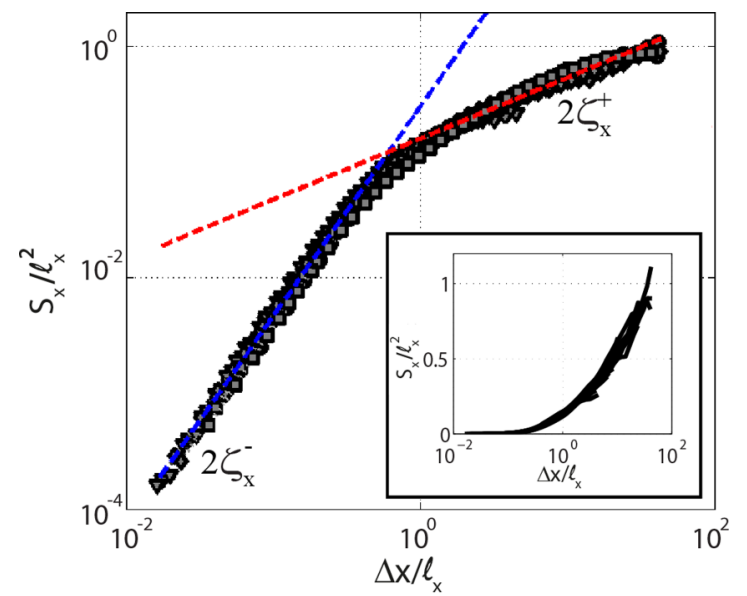

(a)

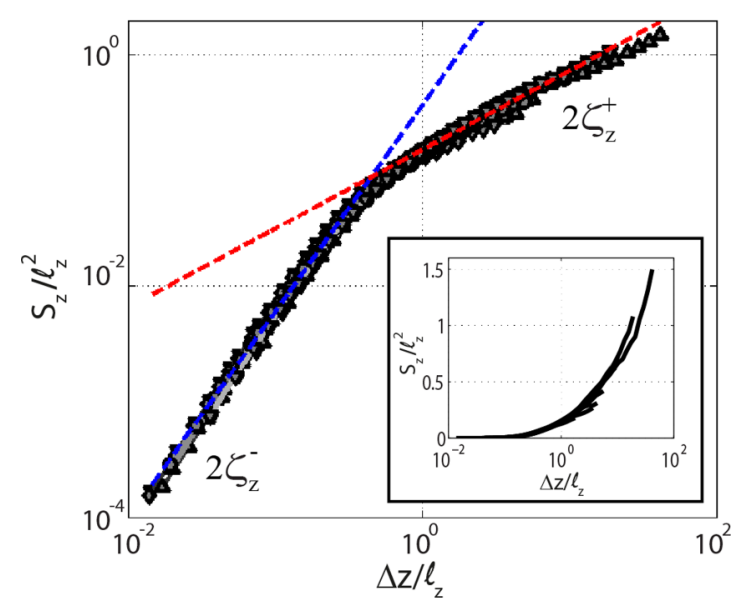

(b)

FIG. 6. (Color online) Structure functions normalized by the optimal lengths $\ell_{x}$ and $\ell_{z}$. Measurements performed along (a) $x$ (propagation direction) and (b) $z$ (front crack). Both axes are logarithmic in the main panels and semilogarithmic in the insets. Straight lines in the main panels are power-law fits with exponents reported in Table III.

values of power-law behaviors, the insets in Fig. 6 clearly prove that they are finite and exclude a logarithmic behavior.

\section{Effect of the porosity}

We now investigate the influence of the porosity $\Phi$. Figure 7 shows the dimensionless structure functions. Contrary to the previous case, the data do not collapse. First, the amplitude of the structure functions is found to increase with $\Phi$ at all scales. This is compatible with the observations of rougher

TABLE III. Roughness exponents obtained by linear fits performed on the curves in Fig. 6.

\begin{tabular}{lcc}
\hline \hline & \multicolumn{2}{c}{ Direction } \\
\cline { 2 - 3 } & $x$ & $z$ \\
\hline Small scales: $\zeta^{-}$ & $0.86 \pm 0.04$ & $0.81 \pm 0.05$ \\
Large scales: $\zeta^{+}$ & $0.27 \pm 0.02$ & $0.35 \pm 0.01$ \\
\hline \hline
\end{tabular}

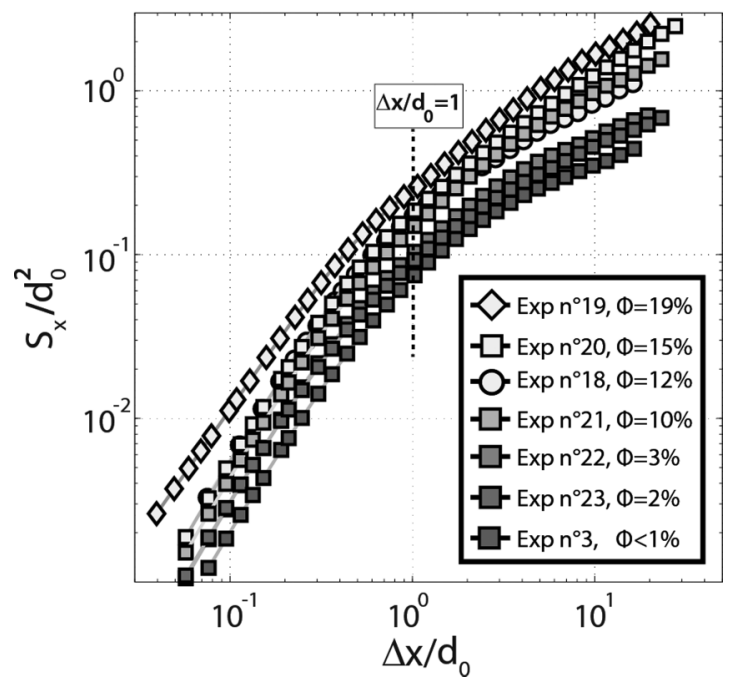

FIG. 7. Dimensionless structure functions along the propagation direction for different porosities $\Phi$.

profiles as the porosity increases (Fig. 4). At small length scales $\left(\Delta x / d_{0}<1\right)$, the exponents are similar for all porosities. A linear regression of the data gives a roughness exponent

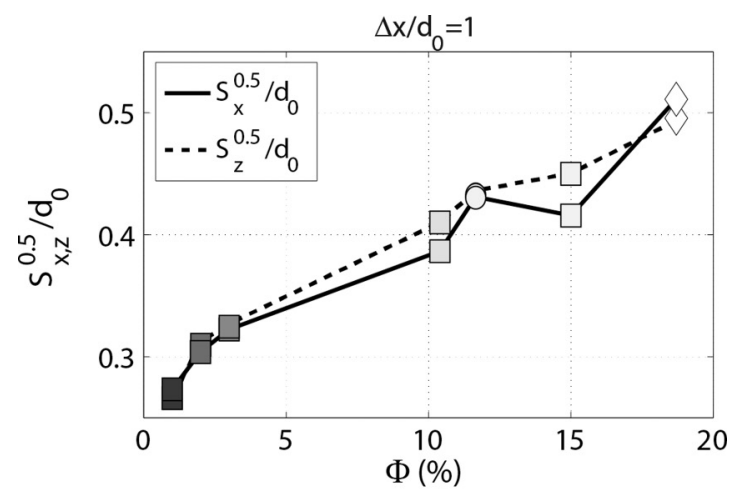

(a)

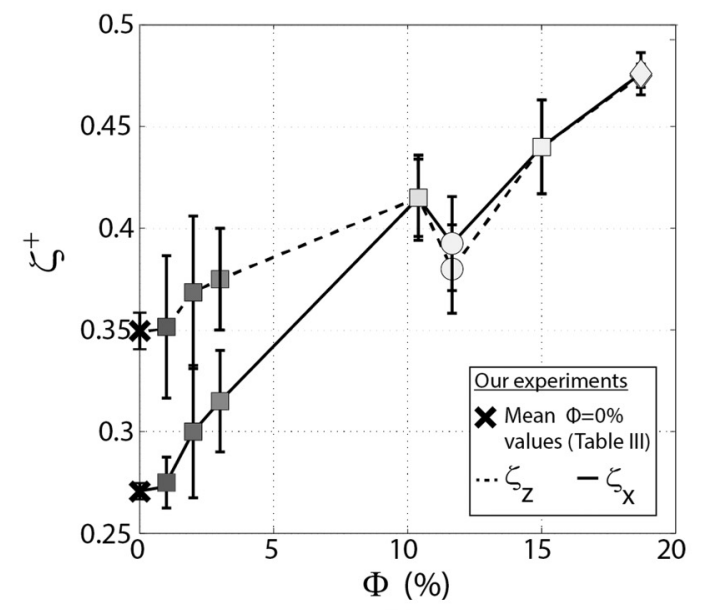

(b)

FIG. 8. (a) Normalized roughness amplitudes in the $x$ and $z$ directions $\sqrt{S\left(d_{0}\right)} / d_{0}$ versus porosity $\Phi$. (b) Large-scale roughness exponents $\zeta^{+}$vs $\Phi$. Symbols match the experiments in Fig. 7. Crosses correspond to the averaged exponent values in Table III. 


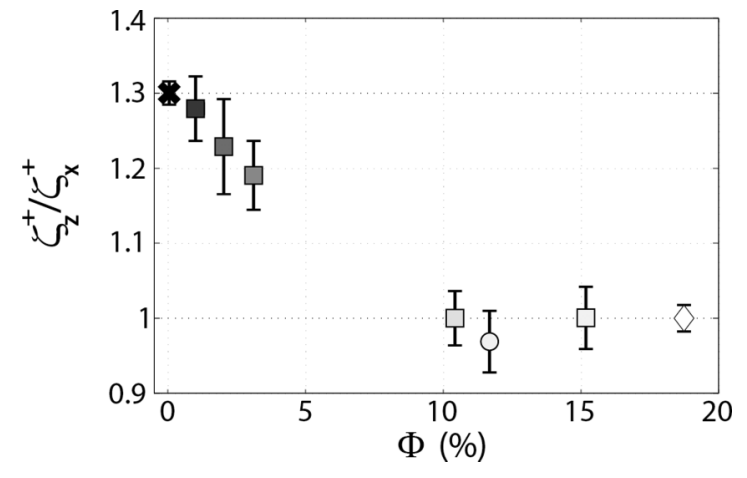

FIG. 9. Scaling anisotropy defined as $\zeta_{z}^{+} / \zeta_{x}^{+}$as a function of the porosity $\Phi$.

$\zeta_{x}^{-}=0.88 \pm 0.04$, very close to that otained for $\Phi=0$. Yet, for $\Delta x / d_{0}>1$, the exponents increase with the porosity.

In Fig. 8, we analyze more quantitatively the effect of $\Phi$ on the roughness. The roughness amplitudes, defined as the value of the structure functions at $\Delta x / d_{0}=1$, are observed to increase linearly with $\Phi$ [Fig. 8(a)]. Figure 8(b) shows the evolution of the large-scale roughness exponents $\zeta_{x}^{+}$and $\zeta_{z}^{+}$ as a function of $\Phi$. As expected, when $\Phi$ tends toward 0 , the values obtained in the no-porosity case (Table III) are recovered. They increase with $\Phi$, up to values of $\zeta_{z}^{+} \sim \zeta_{x}^{+} \sim$ 0.48 for $\Phi=20 \%$. No significant anisotropy is observed in the roughness amplitude [Fig. 8(a)]. Conversely, the scaling anisotropy, i.e., the difference between $\zeta_{x}^{+}$and $\zeta_{z}^{+}$, observed for $\Phi=0$ is recovered. However, it decreases as $\Phi$ increases.

To quantify the scaling anisotropy evolution, we plot the ratio $\zeta_{z}^{+} / \zeta_{x}^{+}$as a function of $\Phi$ in Fig. 9. It decreases from $\zeta_{z}^{+} / \zeta_{x}^{+}=1.3 \pm 0.05$ to 1 as $\Phi$ goes from 0 to $10 \%$. Above, isotropic scaling is obtained.

\section{DISCUSSION}

\section{A. Comparison with other experimental results}

The roughness value $\zeta^{-}$measured at small scales is observed to be independent of the bead size, the direction measurement, and the porosity. It is compatible with the $\zeta=0.8$ value reported in the literature for a variety of materials $[3,5,6]$. Here, $\zeta^{-}$is observed at a scale smaller than the bead size. This small-scale regime is not, strictly speaking, a self-affine regime: The $\zeta^{-}$value can be interpreted as the measured roughness exponent of a piecewise linear profile analyzed at scales smaller than that of its segments (see [44] for a related analysis).

Regarding the roughness properties at large scales, it is interesting to compare our results with what has been reported for ceramics obtained by sintering oxide glass beads [28]. The roughness amplitude [defined as $\sqrt{S\left(\delta r=d_{0}\right)}$ ] is found to increase linearly with $\Phi$ as observed in Ref. [28] for glass ceramics. Conversely, our data converge toward a finite amplitude as $\Phi \rightarrow 0$, while in glass ceramics the amplitude was vanishing. The main difference is that, in Ref. [28], decreasing $\Phi$ induces a transition from an inter- to an intragranular propagation mode, which is not the case here.

The values of the roughness exponents are also, within the error bars, similar to the values of $\zeta \simeq 0.4-0.5$ reported in these glass ceramics [28] and in sandstone [27,30]. Our material is hence more representative of conventional heterogeneous brittle materials such as ceramics and some rocks. As a bonus, the smaller error bars and the larger range of $\Phi$ explored have evidenced the increase in $\zeta$ with $\Phi$. They have also revealed scaling anisotropy in the absence of any porosity, which decreases as $\Phi$ increases and disappears for $\Phi \geqslant 10 \%$.

\section{B. Comparison with theoretical results}

Since the early 1990s, a great deal of theoretical work has focused on the spatial distribution of the fracture surface roughness in brittle heterogeneous materials. They can be classified into two categories: (i) elastic string models, which consider the crack front as an elastic line propagating through randomly distributed obstacles [9-12,14,45]; and (ii) random lattice models, which model the material by a network of fuses, springs, or beams with randomly distributed breakdown thresholds $[8,16,18,46]$. The main difference between the predictions of these two classes of models is that models of type i naturally lead to anisotropic surfaces, where the direction of front propagation plays a specific role, while models of type ii lead to isotropic surfaces [21].

In essence, elastic line models address situations of nominally brittle fracture, with nonporous materials. Hence, they are the relevant theoretical framework in which to discuss the observations reported in Sec. III B. In particular, elastic string approaches lead to

(a) the length scale of the microstructural disorder being the single relevant length scale for the structure function and

(b) anisotropic surfaces, where the direction of front propagation plays a specific role.

Both these predictions are in agreement with the experimental observations (Figs. 6 and 8).

Conversely, the values of the roughness exponents, $\zeta_{x}^{+} \approx$ 0.27 and $\zeta_{z}^{+} \approx 0.35$, observed here are clearly distinct from the values predicted theoretically. In particular, the most refined models $[12,14]$ attempted to derive rigorously the equation of motion of the elastic string from linear elastic fracture mechanics. They yield either logarithmic scaling [12] or $\zeta_{x}=0.50 \pm 0.05$ and $\zeta_{z} \approx 0.385 \pm 0.05$ [14], according to the disorder introduced in the equation. The values reported here are clearly different, which indicates that some key ingredients are missing in the models. In particular, no model takes into account the $T$-stress influence. The $T$ stress here has been determined [47] both by finite-element simulations and digital image correlations [48]. As shown in Fig. 10, the $T$ stress is positive, so that the crack path should be unstable toward any perturbation of its rectilinear path [49]. In other words, additional correlations are anticipated to result from a positive $T$ stress, and hence a larger value for the roughness exponent measured along $x$. If this scenario is correct, it may partly explain the departure from logarithmic behavior.

As mentioned above, in elastic line models, the dimensionless curves $S_{x} / d_{0}^{2}$ vs $\Delta x / d_{0}$ and $S_{z} / d_{0}^{2}$ vs $\Delta z / d_{0}$ are anticipated to be universal. But we observed a scaling function of $\Phi$ (see Fig. 7). This indicates that elastic line approaches stop being relevant as the porosity becomes finite. This is thought to be due to the presence of microcracks forming at the pores, causing the fracture propagation mode to shift gradually 


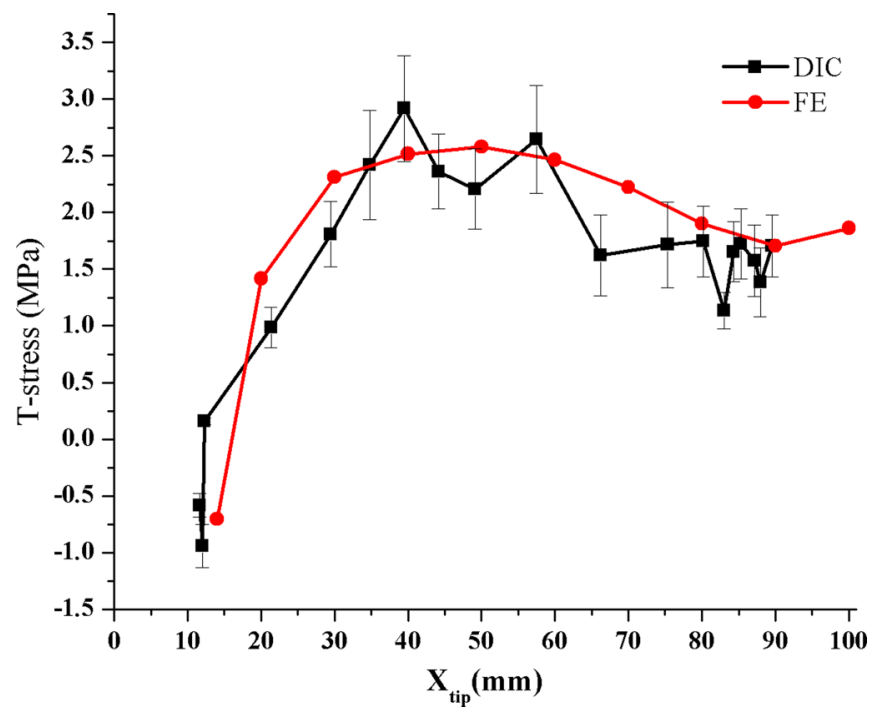

FIG. 10. (Color online) $T$ stress as a function of the crack tip position $x_{\text {tip }}$ obtained by classical finite-element simulations and digital image correlation.

from nominally brittle to quasibrittle. The anisotropy decrease with $\Phi$ is consistent with this scenario: As microcracking develops, the fracture surface stops being the trace left by a single propagating line in an otherwise intact material but results from the coalescence of multiple microcracks instead. Random lattice models include such processes and may be the relevant framework in which to describe this quasibrittle regime. In this context, it is interesting to note that the roughness exponents $\zeta_{x}^{+}=\zeta_{z}^{+} \approx 0.45$ measured here on isotropic fracture surfaces, i.e., above $\Phi=10 \%$, are consistent with the values $\zeta_{\text {loc }}=0.42, \zeta_{\text {loc }}=0.5$, and $\zeta_{\text {loc }}=0.48$ observed, respectively, in three-dimensional random fuse [16], spring [50], and beam [18] networks.

In the above scenario, porosity provides a tunable parameter to go gradually from nominally brittle fracture, where the disorder effect is distortion of the front propagation, to quasibrittle fracture, where the fracture surfaces emerge from a percolating path throughout the microcrack cloud. At present, the theoretical descriptions of these two situations belong to two distinct realms: elastic line models for the former and random lattice models for the latter. Unifying these two frameworks represents an important challenge for future investigations (see [36] for a recent thrust in this direction).

\section{CONCLUSION}

In this paper, we have used homemade cemented grain materials to investigate the influence of the microstructure size and porosity on the fracture surfaces. The following observations have been made:

(a) The roughness displays scale-invariant morphological features that depend on both the grain size and the porosity.

(b) At zero porosity, the structure functions measured along a direction and normalized by the grain size collapse into a single master curve.

(c) Scaling anisotropy is well pronounced for $\Phi=0$ but decreases with $\Phi$ and vanishes for $\Phi>10 \%$.

(d) The values of the roughness exponents increase with $\Phi$, from $\zeta_{x}^{+} \sim 0.27$ and $\zeta_{z}^{+} \sim 0.35$ at $\Phi=0 \%$ to $\zeta_{x}^{+} \sim \zeta_{z}^{+} \sim$ 0.48 at $\Phi=20 \%$.

These observations are compatible with previous studies considering other sintered glass bead materials [28] or sandstone [27,30]. But in these previous studies, the precision was not sufficient to detect any clear variation with $\Phi$. However, the values of the roughness exponents, $\zeta_{x}^{+} \approx 0.27$ and $\zeta_{z}^{+} \approx 0.35$, observed at low porosity are clearly distinct from the values predicted theoretically: Additional investigations are necessary to take into account some missing ingredients like the $T$ stress. Conversely, for $\Phi>10 \%$, the roughness exponents $\zeta_{x}^{+}=\zeta_{z}^{+} \approx 0.45$ are consistent with the values of $\zeta_{\text {loc }}$ between 0.4 and 0.5 obtained with random networks $[16,18,50]$. Modulating $\Phi$ has, thus, permitted us to modify quantitatively the value of the roughness exponents. Note that, in conventional interface growth problems, the roughness exponent value is characteristic of a universality class. Their continuous evolution with $\Phi$ observed herein may pose a rather severe test for current and future competing models of heterogeneous fracture. The $T$-stress component acting on the fracture is controlled by the macroscopic shape of the porous samples; future wors will consider the effect of the $T$ stress on the roughness.

\section{ACKNOWLEDGMENTS}

We acknowledge support by the ANR Mephystar (Grant No. ANR-09-SYSC-006-01). We acknowledge P. Viel for the use of the Bruker profilometer and T. Bernard for technical support. We thank R. Pidoux, L. Auffray, and T. Bernard for realizing the samples.
[1] D. Hull, Fractography: Observing, Measuring and Interpreting Fracture Surface Topography (Cambridge University Press, Cambridge, 1999).

[2] B. B. Mandelbrot, D. E. Passoja, and A. J. Paullay, Fractal character of fracture surfaces of metals, Nature 308, 721 (1984).

[3] E. Bouchaud, Scaling properties of cracks, J. Phys.: Condensed Matter 9, 4319 (1997).
[4] D. Bonamy and E. Bouchaud, Failure of heterogeneous materials: A dynamic phase transition? Phys. Rep. 498, 1 (2011).

[5] E. Bouchaud, G. Lapasset, and J. Planès, Fractal dimension of fractured surfaces: A universal value? Europhys. Lett. 13, 73 1990.

[6] K. J. Måløy, A. Hansen, E. L. Hinrichsen, and S. Roux, Experimental measurements of the roughness of brittle cracks, Phys. Rev. Lett. 68, 213 (1992). 
[7] J. Schmittbuhl, S. Gentier, and S. Roux, Field measurements of the roughness of fault surfaces, Geophys. Res. Lett. 20, 639 (1993).

[8] A. Hansen, H. Hinrichsen, and S. Roux, Roughness of crack interfaces, Phys. Rev. Lett. 66, 2476 (1991).

[9] J. P. Bouchaud, E. Bouchaud, G. Lapasset, and J. Planès, Models of fractal cracks, Phys. Rev. Lett. 71, 2240 (1993).

[10] J. Schmittbuhl, S. Roux, J. P. Vilotte, and K. J. Måløy, Interfacial crack pinning: Effect of nonlocal interactions, Phys. Rev. Lett. 74, 1787 (1995).

[11] H. Larralde and R. C. Ball, The shape of slowly growing cracks, Europhys. Lett. 30, 87 (1995).

[12] S. Ramanathan, D. Ertas, and D. S. Fisher, Quasistatic crack propagation in heterogeneous media, Phys. Rev. Lett. 79, 873 (1997).

[13] A. Hansen and J. Schmittbuhl, Origin of the universal roughness exponent of brittle fracture surfaces: Stress weighted percolation in the damage zone, Phys. Rev. Lett. 90, 045504 (2003).

[14] D. Bonamy, L. Ponson, S. Prades, E. Bouchaud, and C. Guillot, Scaling exponents for fracture surfaces in homogeneous glass and glassy ceramics, Phys. Rev. Lett. 97, 135504 (2006).

[15] Y. Termonia and P. Meakin, Formation of fractal cracks in a kinetic fracture model, Nature 320, 429 (1986).

[16] V. I. Raisanen, M. J. Alava, and R. M. Nieminen, Fracture of three-dimensional fuse networks with quenched disorder, Phys. Rev. B 58, 14288 (1998).

[17] P. Kumar V. V. Nukala, S. Zapperi, and S. Šimunović, Crack surface roughness in three-dimensional random fuse networks, Phys. Rev. E 74, 026105 (2006).

[18] P. K. V. V. Nukala, P. Barai, S. Zapperi, M. J. Alava, and S. Simunovic, Fracture roughness in three-dimensional beam lattice systems, Phys. Rev. E 82, 026103 (2010).

[19] S. Morel, T. Lubet, J.-L. Pouchou, and J.-M. Olive, Roughness analysis of the cracked surfaces of a face centered cubic alloy, Phys. Rev. Lett. 93, 065504 (2004).

[20] N. Mallick, P-P. Cortet, S. Santucci, S. G. Roux, and L. Vanel, Discrepancy between subcritical and fast rupture roughness: A cumulant analysis, Phys. Rev. Lett. 98, 255502 (2007).

[21] L. Ponson, D. Bonamy, and E. Bouchaud, Two-dimensional scaling properties of experimental fracture surfaces, Phys. Rev. Lett. 96, 035506 (2006).

[22] L. Ponson, D. Bonamy, H. Auradou, G. Mourot, S. Morel, E. Bouchaud, C. Guillot, and J.-P. hulin, Anisotropic self-affine properties of experimental fracture surfaces, Int. J. Fract. 140, 27 (2006).

[23] J. M. Lopez and J. Schmittbuhl, Anomalous scaling of fracture surfaces, Phys. Rev. E 57, 6405 (1998).

[24] S. Morel, J. Schmittbuhl, J. M. Lopez, and G. Valentin, Anomalous roughening of wood fractured surfaces, Phys. Rev. E 58, 6999 (1998).

[25] J. Schmittbuhl, F. Schmitt, and C. Scholz, Scaling invariance of crack surfaces, J. Geophys. Res. 100, 5953 (1995).

[26] S. Santucci, K.-J. Maloy, A. Delaplace A, J. Mathiesen, A. Hansen A, J. O. H. Bakke, J. Schmittbuhl, L. Vanel L, and P. Ray, Statistics of fracture surfaces, Phys. Rev. E 75, 016104 (2007).

[27] F. Plouraboué, K. W. Winkler, L. Petit, J. P. Hulin, and S. Roux, Experimental study of fracture surface roughness on rocks with crack velocity, Phys. Rev. E 53, 277 (1996).
[28] L. Ponson, H. Auradou, P. Vié, and J.-P. Hulin, Low self-affine exponents of fractured glass ceramics surfaces, Phys. Rev. Lett. 97, 125501 (2006)

[29] E. Bouchbinder, I. Procaccia, S. Santucci, and L. Vanel, Fracture surfaces as multiscaling graphs, Phys. Rev. Lett. 96, 055509 (2006)

[30] L. Ponson, H. Auradou, M. Pessel, V. Lazarus, and J.-P. Hulin, Failure mechanisms and surface roughness statistics of fractured fontainebleau sandstone, Phys. Rev. E 76, 036108 (2007).

[31] D. Dalmas, A. Lafarge, and D. Vandembroucq, Crack propagation through phase separated glasses: Effect of the characteristic size of disorder, Phys. Rev. Lett. 101, 255501 (2008).

[32] E. Bouchaud, D. Boivin, J.-L. Pouchou, D. Bonamy, B. Poon, and G. Ravichandran, Fracture through cavitation in a metallic glass, Europhys. Lett. 83, 66006 (2008).

[33] L. Ponson, Y. Cao, E. Bouchaud, V. Tvergaard, and A. Needleman, Statistics of ductile fracture surfaces: The effect of material parameters, Int. J. Fract. 184, 137 (2013).

[34] A. Srivastava, L. Ponson, S. Osovski, E. Bouchaud, V. Tvergaard, and A. Needleman, Effect of inclusion density on ductile fracture toughness and roughness, J. Mech. Phys. Solids 63, 62 (2014).

[35] S. Morel, D. Bonamy, L. Ponson, and E. Bouchaud, Transient damage spreading and anomalous scaling in mortar crack surfaces, Phys. Rev. E 78, 016112 (2008).

[36] K. S. Gjerden, A. Stormo, and A. Hansen, Universality classes in constrained crack growth, Phys. Rev. Lett. 111, 135502 (2013).

[37] L. Ponson, D. Bonamy, and L. Barbier, Cleaved surface of i-alpdmn quasicrystals: Influence of the local temperature elevation at the crack tip on the fracture surface roughness, Phys. Rev. B 74, 184205 (2006).

[38] J. Barés, Failure in brittle heterogeneous materials: Intermittency, crackling and seismicity, Ph.D. thesis, Ecole Polytechnique, Paris, 2013.

[39] J. Scheibert, C. Guerra, F. Célarié, D. Dalmas, and D. Bonamy, Brittle-quasibrittle transition in dynamic fracture: An energetic signature, Phys. Rev. Lett. 104, 045501 (2010).

[40] C. Guerra, J. Scheibert, D. Bonamy, and D. Dalmas, Understanding fast macroscale fracture from microcrack post mortem patterns, Proc. Natl. Acad. Sci. USA 109, 390 (2012).

[41] H. A. Janssen, Versuche ber getreidedruck in silozellen, Z. Vereines Deutsch. Ingen. 39, 1045 (1895).

[42] D. Bonamy, Intermittency and roughening in the failure of brittle heterogeneous materials, J. Phys. D: Appl. Phys. 42, 214014 (2009).

[43] T. Cambonie, N. Gautier, and J. L. Aider, Experimental study of counter-rotating vortex pair trajectories induced by a round jet in cross-flow at low velocity ratios, Exp. Fluids 54, 1 (2013)

[44] F. Lechenault, G. Pallares, M. George, C. L. Rountree, E. Bouchaud, and M. Ciccotti, Effects of finite probe size on self-affine roughness measurements, Phys. Rev. Lett. 104, 025502 (2010).

[45] E. Katzav, M. Adda-Bedia, and B. Derrida, Fracture surfaces of heterogeneous materials: A 2D solvable model, Europhys. Lett. 78, 46006 (2007) 
[46] L. de Arcangelis, A. Hansen, H. J. Herrmann, and S. Roux, Scaling laws in fracture, Phys. Rev. B 40, 877 (1989).

[47] L. Hattali, H. Auradou, M. Francois, and V. Lazarus, Evaluation using digital image correlation and finite element method of stress intensity factors and $\mathrm{t}$-stress in wedge splitting test, in The 13rd International Conference on Fracture (ICF-13), Beijing, 16-21 June (International Congress on Fracture (ICF), China, 2013).
[48] F. Mathieu, F. Hild, and S. Roux, Identification of a crack propagation law by digital image correlation, Int. J. Fatigue 36, 146 (2012).

[49] B. Cotterell and J. R. Rice, Slightly curved or kinked cracks, Int. J. Fract. 16, 155 (1980).

[50] A. Parisi, G. Caldarelli, and L. Pietronero, Roughness of fracture surfaces, Europhys. Lett. 52, 304 (2000). 